\title{
Sintomas Depressivos em Crianças: Estudos com duas Versões do CDI
}

Depressive symptoms in children: Studies with two versions of CDI

Síntomas depresivos en niños: Estudios con dos versiones del CDI
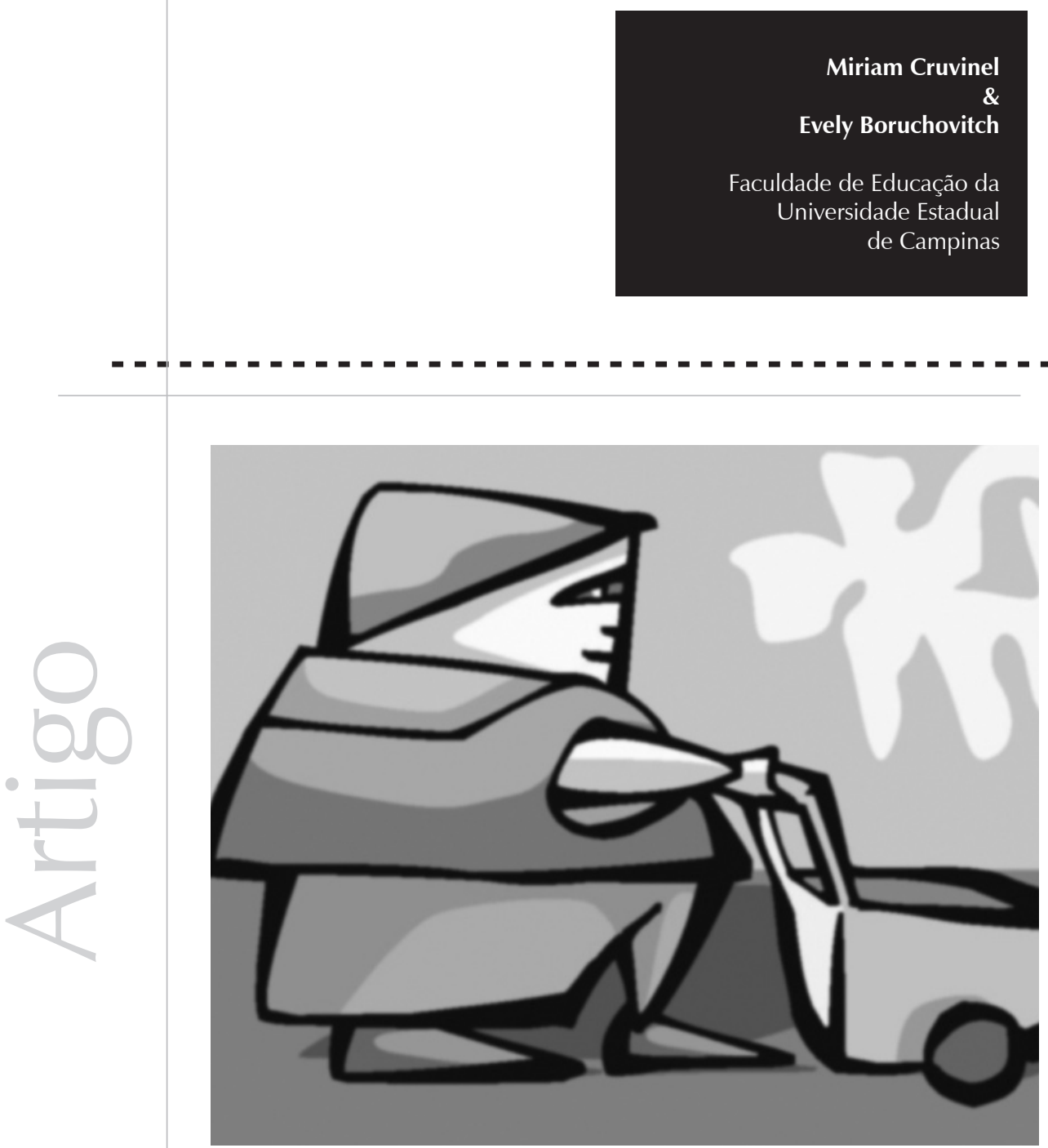
Resumo: O objetivo deste estudo foi identificar o número de crianças com sintomas depressivos em duas amostras de estudantes da cidade de Campinas. Utilizou-se, no estudo 1, a versão brasileira do Inventário de Depressão Infantil (CDI) e, no estudo 2, a versão original. Do estudo 1, realizado em 2001, participaram

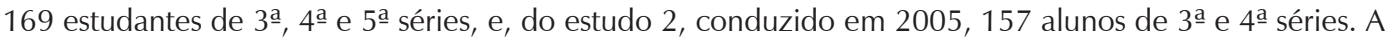
prevalência de sintomatologia depressiva no estudo 1 foi de 3,55\%, e, no estudo 2, de 17\%. Em ambos os estudos, não foram encontradas diferenças significativas entre a média no CDI em relação às variáveis demográficas. Conclui-se pela necessidade de mais estudos nacionais de prevalência no ensino fundamental e de esforços direcionados não só para a identificação dos fatores de risco, de proteção e das variáveis associadas mas também do refinamento dos instrumentos de medidas da depressão infantil.

Palavras-chave: Depressão infantil. Avaliação psicológica. Inventário de Depressão Infantil. Educação básica.

\begin{abstract}
The objective this study was to identify the prevalence of depressive sintomatology in two samples of public school basic education students of Campinas. Two instrumennts were employed: the validated and reduced version of the Child Depressive Inventory (CDI) and its original and translated version. The sample consisted of 169 from $3^{\text {rd }}, 4^{\text {th }}$ and $5^{\text {th }}$ grades (study 1 ) and of 157 from $3^{\text {rd }}$ and $4^{\text {th }}$ grades (study 2). The prevalence of depressive sintomatology was 3, 55\% in study 1 and $17 \%$ in study 2 . No significant relationships emerged between demographic variables (age, gender, school grade, school retention) and depressive sintomatology in both studies As an increase in the rates of prevalence of this sintomatology has been evinced, there is a need for investigations with larger samples of basic education students, for directing efforts towards identifying risk and protection factors associated with the development of this disease, and towards refining its measurement instruments.
\end{abstract}

Keywords: Child Depression. Psychological Evaluation. Child Depression Inventory. Basic Education.

Resumen: El objetivo de este estudio fue identificar el número de niños con síntomas depresivos en dos muestras de estudiantes de la ciudad de Campinas. Se utilizó, en el estudio 1, la versión brasileña del Inventario de Depresión Infantil (CDI) y, en el estudio 2, la versión original. Del estudio 1, realizado en 2001, participaron 169 estudiantes de 3aㅡ, $4^{\underline{a}}$ y $5^{\underline{a}}$ series, y, del estudio 2, dirigido en 2005, 157 alumnos de $3^{\underline{a}}$ y 4 a series. La permanencia de sintomatología depresiva en el estudio 1 fue de 3,55\%, y, en el estudio 2, de $17 \%$. En ambos estudios, no fueron encontradas diferencias significativas entre la media en el CDI con relación a las variables demográficas. Se concluye por la necesidad de más estudios nacionales de superioridad en la enseñanza fundamental y de esfuerzos dirigidos no sólo para la identificación de los factores de riesgo, de protección y de las variables asociadas sino también del refinamiento de los instrumentos de medidas de la depresión infantil.

Palabras-clave: Depresión infantil. Evaluación psicológica. Inventario de Depresión Infantil. Educación básica.

A depressão infantil tem merecido destaque, nos últimos anos. Nota-se que, com o aumento crescente das taxas de prevalência de depressão na população em geral, o problema passou a ser reconhecido na literatura, o que contribuiu para a ampliação e a divulgação de estudos acerca do tema, principalmente, em nosso meio (Cambaúva \& Silva Jr., 2005; Monteiro \& Lage, 2007). Em linhas gerais, têm-se constatado controvérsias nos resultados encontrados nas investigações a respeito do tema. Apesar disso, os estudiosos concordam com o fato de que a depressão traz uma série de prejuízos à vida diária da criança e do adolescente, prejudicando seriamente suas relações familiares e sociais (Fu I, Curatolo, \& Friedrich, 2000; Harrington, 1993; Monteiro, Coutinho, \& Araújo, 2007).

Sabe-se que a criança que possui sintomas depressivos apresenta características próprias quanto ao funcionamento psicológico. Normalmente, a depressão, na infância, vem associada à baixa auto-estima, ao isolamento social, à queda no rendimento 
escolar, ao retraimento e à labilidade emocional (Bahls, 2002; Stevenson \& Romney, 1984). Goodman, Schwab-Stone, Lahey, Schaffer e Jensen (2000) observaram que problemas com a família e dificuldades de relacionamento com os pais, muitas vezes, caracterizam o contexto familiar de crianças com diagnóstico de depressão. Já na adolescência, são freqüentes graves alterações de comportamento, especialmente o uso abusivo de álcool e drogas. Corroborando dados de investigações anteriores, Monteiro et al. (2007), ao avaliarem grupos de adolescentes com e sem sintomatologia depressiva, verificaram que adolescentes com sintomas de depressão tendem a descrever a depressão como algo que poderia levá-los ao uso de drogas. Solidão, sofrimento, raiva e angústia foram as principais associações dos participantes à depressão.

No que concerne à taxa de prevalência de depressão na infância e na adolescência, ainda há resultados contraditórios. Miller (2003) descreve que $2,5 \%$ das crianças em idade escolar e 8,5\% dos adolescentes apresentam transtornos depressivos. Diversos estudos encontraram índices mais altos de prevalência de sintomatologia depressiva na infância (Colbert, Newman, Ney, \& Young, 1992; Culp, Clyman, \& Culp, 1995; Kleftaras \& Didaskalou, 2006). Na investigação de Colbert et al. (1992), foi observada uma taxa de $54 \%$ de crianças, que residiam no oeste do Canadá, entre 6 a 14 anos de idade, com sintomas de depressão, sendo que os meninos apresentaram mais sintomas depressivos que as meninas. Resultados semelhantes foram vistos em Culp et al. (1995), no qual foram encontrados $57 \%$ de crianças e adolescentes dos EUA, entre 11 a 18 anos, com sintomatologia depressiva. Já na pesquisa de Kleftaras e Didaskalou (2006), realizada com estudantes de $3^{\underline{a}}$ e $5^{\underline{a}}$ séries, na Grécia, cerca de $30 \%$ da amostra apresentou pontuação elevada no CDI.
No Brasil, apesar da escassez de estudos epidemiológicos, os resultados de algumas investigações têm revelado que, de fato, a depressão ocorre na infância. Bahls (2002) encontrou $20,3 \%$ de estudantes com idade entre 10 e 17 anos, da cidade de Curitiba, que ultrapassaram o ponto de corte 19 no CDI. Fonseca, Ferreira e Fonseca (2005) observaram que $13,7 \%$ de escolares de 7 a 13 anos de idade, de Minas Gerais, apresentaram uma pontuação superior a 17 no CDI. Níveis altos de sintomatologia depressiva foram encontrados entre os adolescentes avaliados no estudo de Rocha, Ribeiro, Pereira, Aveiro e Silva (2006). Empregando o questionário SRQ-20 - self reporting questionnaire, um instrumento desenvolvido pela Organização Mundial da Saúde, os resultados apontaram a presença de sintomas depressivos em $45,7 \%$ dos adolescentes mineiros, sendo que a prevalência foi duas vezes maior no sexo feminino.

Outras investigações conduzidas em diversas regiões do Brasil sugerem índices menores de prevalência de sintomas depressivos. Hallak (2001), por exemplo, encontrou, em Ribeirão Preto, 6\% de escolares com altos escores no CDI e 3\% ao empregar a Escala de Sintomatologia Depressiva para Professores (ESDM-P). Também em Ribeirão Preto, Baptista e Golfeto (2000) verificaram que 1,48\% da amostra de crianças e adolescentes tiveram um escore elevado no CDI. Já no estudo de Soares (2003), conduzido no Rio Grande do Sul, foi encontrada uma prevalência de 2,39\% de sintomas de depressão mediante a aplicação do CDI.

Em recente revisão a respeito das taxas de prevalência de maior depressão em crianças e adolescentes, Bahls (2002) revelou que o 
índice para crianças varia de 0,4 a 3,0\%, e, para os adolescentes, de 3,3 a 12,4\%.

Conclui-se pela literatura nacional e internacional que o número de crianças com o problema é bastante variável. De acordo com Poznanski e Mokros (1994), são inúmeros os fatores que contribuem para a variação nos índices de depressão infantil. Diferenças na população estudada, nos métodos de avaliação, no diagnóstico de depressão e diferenças no ponto de corte dos instrumentos são variáveis que interferem nos resultados de prevalência. Além disso, Baptista e Golfeto (2000) lembram ainda que as diferenças regionais, econômicas e culturais na população brasileira podem também contribuir para a diversidade dos resultados encontrados. No entanto, vale ressaltar que as variações nas taxas de prevalência continuam mesmo quando os diversos estudos usam instrumentos de medidas de depressão semelhantes. Estudos que utilizaram o Inventário de Depressão Infantil - CDI (Kovács, 1992) no Brasil para a identificação de sintomas depressivos oferecem dados divergentes. Alguns autores brasileiros (Bahls, 2002; Fonseca et al., 2005) encontraram índices de prevalência semelhantes, que variaram de $13,7 \%$ a $22 \%$. Já outros (Baptista \& Golfeto, 2000; Hallak, 2001; Soares, 2003) obtiveram taxas de prevalência diferentes, que se dispersaram entre 1,48\% a $6 \%$. Vale dizer que, Timbremont, Braet e Dreessen (2004) examinaram a utilidade do CDI no diagnóstico para depressão maior e a adequação do ponto de corte. Os resultados revelaram que o Inventário é eficiente na identificação da depressão, além de diferenciar a depressão de outras desordens como ansiedade e problemas de conduta. Os autores concluíram também que o ponto de corte de 16 apresentou ótima relação entre especificidade e sensibilidade na identificação da depressão.

\section{Prevalência de depressão na infância em relação ao gênero e idade}

A literatura aponta também para divergências nos dados de prevalência de sintomas depressivos no que concerne ao gênero e à idade. Parece que, a partir da adolescência, as meninas passam a apresentar mais sintomas de depressão do que os meninos. Antes da adolescência, esses índices ainda são confusos, no entanto, quando os estudos apontam o fato de que meninas são mais depressivas que os meninos, algumas hipóteses são levantadas, e uma delas refere-se, segundo Harrington (1993), às formas de socialização. Esse autor explica que, normalmente, as meninas lidam com suas emoções de forma diferente dos meninos. As meninas são encorajadas a entrar em contato com sentimentos depressivos como tristeza, choro e desânimo, e os meninos são reforçados a utilizar outras estratégias, como, por exemplo, a distração.

Quanto à prevalência de sintomas depressivos, de acordo com a idade, tem se verificado que, na medida em que a idade se aproxima da adolescência, há um aumento no número de casos. Apesar disso, existem estudos que encontraram resultados diferentes. Há investigações que não verificaram relações significativas entre sintomas de depressão e faixa etária (Fonseca et al., 2002; Gouveia, Barbosa, Almeida, \& Gaião, 1995). Alguns autores explicam que a elevada taxa de prevalência de depressão a partir da adolescência parece acompanhar o desenvolvimento cognitivo e emocional. Nessa fase, ocorre maior maturidade cognitiva, o que permite que o adolescente seja mais reflexivo, introspectivo e autocrítico, habilidades essas necessárias para o aparecimento de crenças e de avaliações negativas de si 
mesmo e do mundo. Além desses fatores, a adolescência é caracterizada pela confusão e pela presença de conflitos e pela busca de identidade e de novos relacionamentos, o que, por si só, já poderia contribuir para o desenvolvimento da depressão no adolescente (Baptista, 1997). Outros fatores que parecem estar associados ao fato de crianças menores apresentarem menos depressão referem-se ao suporte social e à presença de eventos estressores. As crianças tendem a vivenciar menos situações de conflito e são mais protegidas quanto aos fatores de risco, além de poderem contar com melhor suporte familiar do que os adolescentes (Harrington, 1993).

Além da discussão de aspectos sociais e psicológicos que interferem na prevalência, as hipóteses biológicas também são consideradas, tendo-se em vista que as diferenças nas taxas de depressão coincidem com a puberdade, período que se caracteriza por grandes transformações hormonais e bioquímicas. Baptista (1997) explica que, na puberdade, surgem alterações hormonais que podem ocasionar mudanças de humor. Para o autor, o início do ciclo menstrual e fases como a gestação podem funcionar como gatilho para o desenvolvimento de episódios depressivos.

Apesar das controvérsias e dificuldades nos estudos a respeito da depressão infantil bem como acerca das variáveis a ela associadas, Birmaher et al. (1996) concluem que existe um aumento, nos últimos anos, da prevalência de depressão em crianças e adolescentes, e sugerem que a depressão parece ocorrer em crianças cada vez mais jovens. Os autores alertam para a necessidade de se identificar precocemente essas crianças a fim de que sejam realizados tratamentos para esses problemas. Nesse sentido, o presente artigo tem como objetivo avaliar a prevalência de sintomatologia depressiva em alunos do ensino fundamental, mediante a aplicação de duas versões do Inventário de Depressão Infantil em dois diferentes estudos. No estudo 1, realizado em 2001, empregouse o Inventário de Depressão Infantil validado e adaptado para a população brasileira por Gouveia et al. (1995). O estudo 2, conduzido em 2005, utilizou o Inventário de Depressão Infantil na sua forma original, com 27 itens, desenvolvido por Kovács (1992) e traduzido para o português por Baptista (1997). Os estudos 1 e 2 serão descritos a seguir.

\section{Método}

\section{Estudo 1}

Participantes

O estudo 1 foi desenvolvido em uma escola municipal da cidade de Campinas, São Paulo, que atendia uma população de nível socioeconômico desfavorecido. A amostra foi composta de 169 crianças de $3^{a}$ série $(\mathrm{N}=69), 4^{a}$ série $(\mathrm{N}=69)$ e de $5^{a}$ série $(\mathrm{N}=$ 31) do ensino fundamental. Os alunos eram de ambos os sexos, sendo que $58 \%$ eram do sexo masculino e $41 \%$ do sexo feminino. A faixa etária dos participantes variou de 8 a 15 anos, prevalecendo um número maior de crianças na idade de 8 a 11 anos. Quanto à história escolar, a maioria dos alunos (73\%) não havia repetido nenhuma série anterior, e $26 \%$ da amostra já havia passado por uma experiência de repetência escolar.

Embora a maioria dos alunos matriculados tenha participado da pesquisa, houve um número acentuado de crianças cujos pais não autorizaram a participação. Cerca de 231 alunos estavam matriculados na $3^{a}, 4^{a}$ 
e $5^{\mathrm{a}}$ séries. Dos alunos matriculados, $84,3 \%$ da $3^{a}$ série, $75,8 \%$ da $4^{a}$ série e $55 \%$ da $5^{a}$ série foram avaliados. O restante dos alunos não foram avaliados, ou em razão de seus pais não autorizarem, ou por não terem devolvido o termo de autorização. Cerca de $8,9 \%$ dos pais dos alunos de $3^{a}$ série, $8,8 \%$ de $4^{a}$ série e $9 \%$ dos de $5^{a}$ série não permitiram que seus filhos participassem da pesquisa. Aproximadamente 35\% dos alunos de $5^{\mathrm{a}}$ série se esqueceram de entregar o termo de autorização para o pesquisador, contrastando com apenas 3,8\% dos alunos de $3^{a}$ série e $15,4 \%$ de $4^{a}$ série.

\section{Instrumento}

No estudo 1, foi empregada a versão do Inventário de Depressão Infantil (CDI) (Kovács, 1992) validada para a população brasileira por Gouveia et al. (1995). O CDI consiste em uma escala de auto-avaliação destinada a identificar os sintomas de depressão em pessoas de 7 a 17 anos. Tem sido amplamente utilizado em estudos epidemiológicos nacionais por ser um instrumento econômico, de fácil aplicação e fácil correção, bem como por ser um instrumento já validado para a população brasileira. A versão validada em nosso meio foi reduzida a 20 itens que mensuram os sintomas afetivos, cognitivos e comportamentais da depressão.

Na presente investigação, a versão validada do CDI ficou composta por 19 itens, uma vez que se optou pela retirada do item 9, que mede a intenção suicida. Tal como para Weisz, Thurber, Proffitt e Sweeney (1997), a omissão desse item teve a finalidade de evitar que os participantes pudessem se abalar emocionalmente.

Durante a aplicação desse instrumento, solicita-se ao participante que marque com um $\mathrm{X}$ a alternativa que melhor descreve seu modo de pensar, agir ou sentir, devendo ser assinalada apenas uma única alternativa. Cada item ou questão possibilita a escolha de três alternativas de resposta, que variam de uma escala de 0 (ausência de sintoma) a 2 pontos (sintoma grave). Assim, a pontuação no CDI varia de 0 a 40 pontos.

Exemplo de um item desse inventário: "Eu fico triste de vez em quando; Eu fico triste muitas vezes; Eu estou sempre triste".

Para essa primeira amostra, a avaliação da consistência interna do CDI foi calculada por meio do coeficiente Alpha de Cronbach, obtendo-se o valor de 0.73 , o que indica que esse inventário pode ser um bom instrumento de triagem na identificação de sintomas depressivos.

Para a adaptação do CDI à realidade brasileira, Gouveia et al. (1995) realizaram a tradução do instrumento, a análise fatorial, a avaliação das variáveis sexo, idade, tipo de escola e escolaridade e a elaboração de normas de diagnóstico. Os resultados mostraram que o CDI é um instrumento unifatorial, com aceitáveis parâmetros psicométricos e eficiente na identificação dos sintomas de depressão na infância. Gouveia et al. (1995) definiram que o ponto de corte no CDI seria 17 pontos, de forma que a criança com uma pontuação igual ou superior a 17 pontos deve merecer atenção, uma vez que tal resultado pode indicar um provável depressivo. Neste estudo, devido à retirada do item 9, o ponto de corte foi recalculado e definiu-se 16 como ponto de corte.

Procedimento de coleta de dados

Foi solicitada aos pais ou responsáveis pelas crianças das séries estudadas uma autorização 
por escrito para a participação das mesmas na pesquisa. No Termo de Consentimento, os pais foram orientados a respeito dos objetivos do estudo, do tipo de participação requerida bem como da ausência de prejuízos decorrentes da não adesão à pesquisa. Foram incluídos no presente estudo somente os alunos que entregaram o termo de autorização devidamente assinado pelos pais.

A aplicação do CDI foi realizada em pequenos grupos de, no máximo, quatro crianças e em apenas uma única entrevista conduzida em horário escolar. Os itens do CDI foram gravados em fita cassete, de forma que não existisse a interferência do pesquisador.

As crianças foram orientadas sobre como preencher o inventário e, caso tivessem dúvidas, que levantassem a mão. Em seguida, as questões do CDI foram ouvidas pelos alunos, que tinham uma cópia do instrumento e acompanhavam os itens gravados. Cada criança respondia às questões em seu próprio formulário. Os estudantes utilizaram aproximadamente 15 minutos para responder o instrumento.

\section{Estudo 2}

\section{Participantes}

Conduzido em outra escola de Campinas, a amostra do estudo 2 foi composta por 157 alunos de 3 a e 4 a séries do ensino fundamental, sendo 70 estudantes $(44,6 \%)$ do sexo masculino e $87(55,4 \%)$ do sexo feminino. A faixa etária dos participantes variou de 8 a 12 anos de idade, com idade média de 9 anos e desvio-padrão de 0,81. No que se refere à escolaridade, 85 alunos $(54,1 \%)$ freqüentavam a 3 a série e $72(45,9 \%)$ estavam na $4 \underline{a}$ série. Todos os participantes estudavam em uma escola pública da cidade de Campinas, São Paulo. Quanto à repetência escolar, verificou-se que 146 alunos (93\%) eram não repetentes, e 11 (7\%), repetentes.

Cabe ressaltar que estavam matriculados na 3a série 136 alunos, e, na 4a série, 135, totalizando 271 estudantes. Dessa forma, o Termo de Consentimento foi enviado aos 271 alunos matriculados nas séries estudadas, e, desses termos, foram devolvidos 157, ou seja, $57,9 \%$. Mais especificamente, na 3 a série, 17 alunos (8,8\%) não participaram devido a não autorização dos pais, e 30 alunos (22\%) não devolveram o termo de consentimento ao pesquisador. Na 4 a série, 22 alunos $(16,2 \%)$ não foram autorizados pelos pais e $39(28,8 \%)$ não devolveram o termo de consentimento. No total, $39,7 \%$ dos alunos não participaram da pesquisa, sendo que 39 alunos (14,3\%) não foram autorizados pelos pais e $69(25,4 \%)$ não entregaram o termo para o pesquisador.

Instrumento

Empregou-se, no estudo 2, o Inventário de Depressão Infantil - CDI desenvolvido por Kovács, em 1992, e traduzido para o português por Baptista (1997). Essa versão do CDI é constituída por 27 itens que avaliam os sintomas afetivos, cognitivos e comportamentais da depressão; o escore varia de 0 a 54 pontos. Assim como no estudo 1, na presente investigação, optou-se pela retirada do item 9, que avalia a intenção de suicídio, de forma que o inventário ficou composto por 26 itens no total. O ponto de corte, no estudo 2, foi 17 pontos.

A avaliação da consistência interna dessa versão do Inventário foi também estimada pelo coeficiente Alpha de Cronbach, tendose encontrado um Alpha de 0,80, índice mais elevado que o alcançado no estudo 1 . 
Procedimento de coleta de dados

Após aceitação da escola, foi solicitada aos pais ou responsável pelas crianças uma autorização por escrito que permitisse a participação das mesmas na pesquisa. O Termo de Consentimento foi enviado aos pais de todos os alunos matriculados na 3 a e $4^{a}$ séries. A coleta de dados foi iniciada após o consentimento dos pais, e cabe ressaltar que, assim como no estudo 1, todos os cuidados éticos tomados no estudo 2 basearam-se na Resolução no 196/96 do Ministério da Saúde (1996).

A administração do CDI foi coletiva e conduzida pelo primeiro autor nas salas de aula, com os participantes cujos pais haviam assinado o Termo de Consentimento. Durante a aplicação do inventário, os alunos que os pais não assinaram o Termo de Consentimento foram retirados da sala de aula e conduzidos para uma outra classe pela professora. Restou, em cada classe, uma média de 20 alunos. Os alunos foram orientados quanto ao preenchimento do Inventário, e cada criança tinha uma cópia do instrumento, no qual assinalavam os itens escolhidos na medida em que o pesquisador lia, pausadamente, as questões. A aplicação do CDI teve duração de aproximadamente 20 minutos.

\section{Resultados e discussão}

Estudo 1

Conforme mencionado anteriormente, no estudo 1, foi empregada a versão do CDI adaptada para a população brasileira, tendo sido utilizado o escore 16 como ponto de corte. Dos 169 participantes da amostra total, cerca de 3,55\% atingiram ou ultrapassaram o ponto de corte no CDI.

Os dados referentes à média e ao desvio-padrão do CDI separados pelas variáveis série escolar, gênero e repetência são apresentados na Tabela 1.

Tabela 1. Média e desvio-padrão do CDI por sexo, série escolar, idade e repetência.

\begin{tabular}{|c|c|c|c|c|}
\hline & $\mathrm{N}$ & Porcentagem & Média & Desvio-padrão \\
\hline \multicolumn{5}{|l|}{ Sexo } \\
\hline M & 99 & $58,6 \%$ & 6,14 & 3,86 \\
\hline $\mathrm{F}$ & 70 & $41,4 \%$ & 7,04 & 5,02 \\
\hline \multicolumn{5}{|l|}{ Série } \\
\hline $3^{\underline{a}}$ & 69 & $40,8 \%$ & 6,70 & 4,49 \\
\hline $4^{\underline{a}}$ & 69 & $40,8 \%$ & 6,45 & 4,82 \\
\hline $5^{\underline{a}}$ & 31 & $18,3 \%$ & 6,26 & 3,03 \\
\hline \multicolumn{5}{|l|}{ Idade } \\
\hline 8 a 9 anos & 74 & $43,8 \%$ & 6,58 & 4,60 \\
\hline 10 a 11 anos & 76 & $45,0 \%$ & 6,54 & 4,30 \\
\hline 12 a 15 anos & 19 & $11,2 \%$ & 6,16 & 4,02 \\
\hline \multicolumn{5}{|l|}{ Repetência } \\
\hline Repetente & 45 & $26,6 \%$ & 7,13 & 3,90 \\
\hline Não repetente & 124 & $73,3 \%$ & 6,29 & 4,54 \\
\hline Total & 169 & $100 \%$ & 6,51 & 4,38 \\
\hline
\end{tabular}


Conforme aponta a Tabela 1, a média obtida pelos participantes no CDI foi de 6,51, e o desviopadrão, de 4,38. Os participantes apresentaram no escore do CDI um valor mínimo igual a 0 e um valor máximo igual a 25.

A análise de variância (ANOVA) não indicou diferença estatisticamente significativa entre as médias no $C D I$ das crianças de diferentes séries escolares $[F(2 ; 167)=0,118 ; p=0,888]$ e de diferentes idades $[F(2 ; 167)=0,072 ; p=0,931]$. Os resultados do Teste $t$ também não indicaram diferenças significativas nas médias do CDI entre os sexos ( $t=-1,262 ; p=0,209)$ e entre as médias dos grupos de repetentes e não repetentes $(t=-1,187 ; p=-0,84)$.

Dessa forma, a análise das variáveis demográficas mostrou não haver diferenças significativas quando os resultados foram comparados por sexo, faixa etária, série e repetência. Dados semelhantes foram obtidos em outros estudos brasileiros que também empregaram a versão adaptada do CDI por Gouveia et al. (1995) na identificação de crianças com sintomas de depressão (Baptista \& Golfeto, 2000; Hallak, 2001; Soares, 2003).

\section{Estudo 2}

Como já relatado anteriormente, no estudo 2, a prevalência de sintomatologia depressiva nos alunos do ensino fundamental foi avaliada mediante a versão original do Inventário de Depressão Infantil, empregando o ponto de corte 17. Da amostra total, cerca de $17 \%$ dos participantes apresentaram uma pontuação no CDI superior ou igual a 17 pontos, o que sugere a presença de sintomas de depressão.

Os dados referentes à média e ao desvio-padrão do CDI separados pelas variáveis série escolar, repetência e gênero são apresentados na Tabela 2.

Tabela 2. Média e desvio-padrão dos escores no CDI em relação às variáveis demográficas.

\begin{tabular}{|c|c|c|c|c|}
\hline & $\mathrm{N}$ & Porcentagem & Média & Desvio-padrão \\
\hline \multicolumn{5}{|l|}{ Sexo } \\
\hline Masculino & 70 & $44,5 \%$ & 8,70 & 6,07 \\
\hline Feminino & 87 & $55,4 \%$ & 9,94 & 6,72 \\
\hline \multicolumn{5}{|l|}{ Série } \\
\hline 3ํㅗ série & 85 & $54,1 \%$ & 10,04 & 7,00 \\
\hline 4ํㅗ série & 72 & $45,8 \%$ & 8,63 & 5,69 \\
\hline \multicolumn{5}{|l|}{ Idade } \\
\hline 8 anos & 38 & $21,3 \%$ & 10,29 & 7,88 \\
\hline 9 anos & 81 & $45,5 \%$ & 9,62 & 6,24 \\
\hline a partir de 10 a & 38 & $21,3 \%$ & 8,00 & 5,13 \\
\hline \multicolumn{5}{|l|}{ Repetência } \\
\hline Não repetente & 146 & $92,9 \%$ & 9,29 & 6,53 \\
\hline Repetente & 11 & $7 \%$ & 10,64 & 5,45 \\
\hline Total & 157 & $100 \%$ & 9,39 & 6,45 \\
\hline
\end{tabular}


O aumento crescente das taxas de

prevalência

de sintomas

depressivos

em crianças e

préadolescentes

tem sido

reconhecido na

literatura, o que tem

contribuído para a

ampliação de

estudos acerca

do tema,

principalmente, em nosso meio

(Bahls, 2002;

Calderaro \&

Carvalho, 2005;

Fonseca et al.,

2005; Nakamura

\& Santos, 2007:

Rocha et al., 2006;

Soares, 2003).
Observou-se, nos resultados obtidos, que a média atingida no CDI foi 9,39, e desviopadrão, de 6,45. Os participantes apresentaram um valor mínimo de 0 e um valor máximo igual a 30 pontos no $\mathrm{CDI}$.

Diferenças entre as médias no CDI e as variáveis demográficas como gênero, série escolar e repetência foram investigadas pelo Teste t, e as diferenças entre médias no CDI e a idade foram avaliadas pela análise de variância (ANOVA). O Teste t não revelou diferenças estatisticamente significativas entre as médias no $C D I$ e o gênero ( $t=-1,215 ; p=0,226$ ) e entre as médias no $\mathrm{CDI}$ e a série escolar $(t=-1,392 ; p=0,166)$. Também não foram encontradas diferenças significativas entre as médias no $\mathrm{CDI}$ e os grupos de repetentes e não repetentes ( $t=-0,776 ; p=0,452)$. A análise de variância (ANOVA) não indicou diferenças significativas entre as médias no CDI e as idades $[F(2 ; 155)=1,307 ; p=0,274]$.

Embora tenham sido empregadas duas versões diferentes do CDI, pode-se observar um aumento no número de crianças com sintomas depressivos, com o passar dos anos, quando se considera que os estudos 1 e 2 foram realizados, respectivamente, em 2001 e 2005.

Observa-se que, em relação às variáveis demográficas, os resultados, tanto no estudo 1 como no estudo 2, são bem semelhantes. Diferenças significativas entre sintomas de depressão e sexo, idade, série e repetência escolar não emergiram. Esses dados corroboram os resultados apresentados pela literatura, já que alguns estudos também não encontraram diferenças significativas entre variáveis demográficas e sintomas de depressão (Bahls, 2002; Culp et al., 1995; Fonseca et al., 2005).

\section{Considerações finais}

O aumento crescente das taxas de prevalência de sintomas depressivos em crianças e pré- adolescentes tem sido reconhecido na literatura, o que tem contribuído para a ampliação de estudos acerca do tema, principalmente, em nosso meio (Bahls, 2002; Calderaro \& Carvalho, 2005; Fonseca et al., 2005; Nakamura \& Santos, 2007; Rocha et al., 2006; Soares, 2003).

Por um lado, o aumento da prevalência entre os dois estudos apresentados parece expressivo, pois, de fato, pode revelar que, com o passar do tempo, o número de crianças e jovens com sintomatologia depressiva esteja se elevando. No caso especifico desses dois estudos, foi interessante notar a freqüência acentuada de alunos que não aderiram à pesquisa, não adesão essa que nos remete a alguns questionamentos, como: O que fez com que os pais desses alunos não autorizassem a participação dos filhos na pesquisa? Por que alguns alunos não entregaram a carta de autorização devidamente assinada? De acordo com Fleming e Offord (1990), é preciso cautela na interpretação de dados de prevalência realizados no ambiente escolar, uma vez que, normalmente, as pesquisas não incluem estudantes que faltam freqüentemente ou alunos evadidos. E, certamente, esses fatores que podem camuflar taxas de sintomatologia depressiva devem ser mais bem investigados por pesquisas futuras.

Por outro lado, o aumento da prevalência nos conduz também a uma reflexão acerca dos múltiplos fatores que podem contribuir para a diversidade de freqüência dos sintomas de depressão. É necessário, pois, considerar as diferenças na forma de aplicação do CDI, no número de itens das duas versões, na definição do ponto de corte, que tem variado na literatura brasileira entre 16 a 19 pontos, bem como as diferenças regionais, socioeconômicas e culturais das populações estudadas.

Mais especificamente, os instrumentos empregados no presente estudo são pontos que merecem destaque. Pode-se aventar a hipótese de que talvez o CDI, na sua versão original traduzida, seja mais sensível na identificação de crianças com sintomas de 
depressão do que o CDI na versão reduzida e adaptada para a população brasileira. Cruvinel, Boruchovitch e Santos (2008) verificaram que o CDI, na sua versão original, desenvolvida por Kovács (1992), apresenta melhores índices de consistência interna, e sugerem que isso se deva possivelmente ao maior número de itens (27 itens), quando comparada à versão adaptada para o Brasil, formada por 20 questões. As autoras destacam a necessidade de que novos estudos sejam conduzidos para que ocorra maior aperfeiçoamento do instrumento brasileiro.

Monteiro e Lage (2007) salientam ainda que, atualmente, na concepção de depressão, tem-se negligenciado o papel da vida psíquica em detrimento de um olhar mais organicista. Sem dúvida, de acordo com os autores, esse enfoque se refletirá em questões relacionadas ao seu diagnóstico e tratamento. Ressaltam que é preciso cautela, não só no diagnóstico da depressão, já que nem toda manifestação de tristeza deve ser considerada como patológica, mas também no seu tratamento, que deve ter por base a singularidade da pessoa, e não apenas a sintomatologia apresentada.

Considerando o aumento de crianças com sintomatologia depressiva, concluise pela necessidade de estudos nacionais mais representativos da sua prevalência no ensino fundamental e de esforços que sejam direcionados não só para a identificação dos fatores de risco, de proteção e das variáveis associadas à depressão infantil mas também ao refinamento de seus instrumentos de medida, a fim de que se possa gerar um corpo de conhecimento mais sólido para melhor auxiliar profissionais da área da saúde na prevenção, triagem, diagnóstico e enfrentamento desse problema.

\footnotetext{
Miriam Cruvinel *

Psicóloga, doutoranda em Educação pela Unicamp, Mestre em Psicologia, Desenvolvimento Humano e Educação pela Faculdade de Educação da Unicamp.

Evely Boruchovitch

Psicóloga pela Universidade do Estado do Rio de Janeiro, PhD em Educação pela University of Southern California, Los Angeles, professora Livre-docente do Departamento de Psicologia Educacional da Faculdade de Educação da Unicamp.

Pesquisadora do Conselho Nacional de Desenvolvimento Científico e Tecnológico (CNPq)

Apoio financeiro Capes e CNPq

* Endereço para envio de correspondência:

Rua Barreto Leme, 2540, ap. 702, Cambuí, Campinas CEP: 13025-085

E-mail: miriam@unicamp.br
} 
Bahls, C. S. (2002). Epidemiology of depressive symptoms in adolescents of a public school in Curitiba, Brazil. Revista Brasileira de Psiquiatria, 24(2), 63-67.

Baptista, M. N. (1997). Depressão e suporte familiar: perspectivas de adolescentes e suas mães. Dissertação de Mestrado em Psicologia, Pontifícia Universidade Católica de Campinas, Campinas, SP.

Baptista, C. A., \& Golfeto, J. H. (2000). Prevalência de depressão em escolares de 7 a 14 anos. Revista de Psiquiatria Clínica, 27(5), 253-255.

Birmaher, B., Ryan N. D., Williamson D., Brent D. A., Kaufman, J., \& Dahl R. E. (1996). Childhood and adolescent depression: A review of the past 10 years. Part I. Journal of the American Academy of Child \& Adolescent Psychiatry, 35(11), 14271439.

Calderaro, R. S. S., \& Carvalho, C. V. (2005). Depressão na infância: um estudo exploratório. Psicologia em Estudo, 10(2), 181-189.

Cambaúva, L. G, \& Silva Jr., M. C. da. (2005). Depressão e neoliberalismo: constituição da saúde mental na atualidade. Psicologia: Ciência e Profissão, 25(4), 526-535.

Colbert, P., Newman, B., Ney, P., \& Young, J. (1992). Learning disabilities as a symptom of depression in children. Journal of Learning Disabilities, 15(6), 333-336.

Cruvinel, M., Boruchovitch, E., \& Santos, A. A. A. (2008). Inventário de depressão infantil (CDI): análise dos parâmetros psicométricos. Revista do Departamento de Psicologia (UFF), 20(2)

Culp, A M., Clyman, M. M., \& Culp, R. E. (1995). Adolescent depressed mood, reports of suicide attempts and asking for help. Adolescence, 30(120), 827-838.

Fleming, J. E., \& Offord, D. R. (1990). Epidemiology of childhood depressive disorders: A critical review. Journal of the American Academy of Child \& Adolescent Psychiatry, 29, 571-580.

Fonseca, A. C., Ferreira, A G., Rebelo, J. A., Simões, M. D. F., Pires, C. L., \& Gregorio, M. H. (2002). O estudo da depressão em crianças: a escala de auto-avaliação da depressão de Birleson. Psychologica, 29, 113-122.

Fonseca, M. H. G., Ferreira, R. A., \& Fonseca, S. G. (2005). Prevalência de sintomas depressivos em escolares. Pediatria, 27(4), 223-232.

Fu I, L., Curatolo, E., Friedrich, S. (2000). Transtornos afetivos. Revista Brasileira de Psiquiatria, 22(Supl. 2), 24-27.

Goodman, S. H., Schwab-Stone, M., Lahey, B. B., Schaffer, D., \& Jensen, P. S. (2000). Major depression and dysthimia in children and adolescents: Discriminant validity and differential consequences in a community sample. Journal of the American Academy of Child and Adolescent Psychiatry, 39(6), 761-770.

Gouveia, V. V., Barbosa, G. A., Almeida, H. J. F., \& Gaião, A. A. (1995). Inventário de depressão infantil - CDI: Estudo de adaptação com escolares de João Pessoa. Jornal Brasileiro de Psiquiatria, 44, 345-349.
Hallak, L. R. L. (2001). Estimativa da prevalência de sintomas depressivos em escolares da rede pública de Ribeirão Preto. Dissertação de Mestrado em Medicina, Universidade de São Paulo, Ribeirão Preto, SP.

Harrington, R. (1993). Depressive disorder in children and adolescents. British Journal of Hospital Medicine, 43, 108112.

Kleftaras, G., \& Didaskalou, E. (2006). Incidence and teachers' perceived causation of depression in primary school children in Greece. School Psychology International, 27(3), 296-314.

Kovács, M. (1992). Children depression inventory CDI: manual. New York: Multi-Health Systems.

Miller, J. (2003). O livro de referência para a depressão infantil (M. M. Tera, trad.). São Paulo: M. Books.

Ministério da Saúde. (1996, 10 de outubro). Resolução no 196/96 do Conselho Nacional de Saúde/MS. Sobre Diretrizes e Normas Regulamentadoras de Pesquisa envolvendo seres humanos. Diário Oficial da União.

Monteiro, F. R., Coutinho, M. P. L., \& Araujo, L. F. (2007). Sintomatologia depressiva em adolescentes do ensino médio: um estudo das representações sociais. Psicologia: Ciência e Profissão, 27(2), 224-235.

Monteiro, K. C. C., \& Lage, A. M. V. (2007). Depressão: uma psicopatolologia classificada nos manuais de psiquiatria. Psicologia: Ciência e Profissão, 27(1), 106-119.

Nakamura, E., \& Santos, J. Q. (2007). Depressão infantil: abordagem antropológica. Revista de Saúde Pública, 41(1), 53-60.

Poznanski, E. O., \& Mokros, H. B. (1994). Phenomenology and epidemiology of mood disorders in children and adolescents. In W. M. Reynolds, Handbook of depression in children and adolescents (pp. 19-40). New York: Plenum Press.

Rocha, T. H. R., Ribeiro, J. E. C., Pereira, G. A., Aveiro, C. C., \& Silva, L. C. A. (2006). Sintomas depressivos em adolescentes de um colégio particular. Psico USF, 11(1), 95-102.

Soares, M. U. (2003). Estudos das variáveis do desenvolvimento infantil em crianças com e sem o diagnóstico de depressão. Tese de Doutorado em Ciência do Movimento Humano, Universidade Federal de Santa Maria, RS.

Stevenson, D. T., \& Romney, D. M. (1984). Depression in learning disabled children. Journal of Learning Desabilities, 17(10), 579-582.

Timbremont, B., Braet, C., \& Dreessen, L. (2004). Assessing depression in youth: Relation between the children's depression inventory and a strutured interview. Journal of Clinical Child and Adolescent Psychology, 33(1), 149-157.

Weisz, J. R., Thurber, C. A, Proffitt, V. D, Sweeney, L., \& Legagnoux, G. L. (1997). Brief treatment of mild-to-moderate child depression using primary and secondary control enhancement training. Journal of Consulting and Clinical Psychology, 65(4), 703-707. 